\title{
Cavitation dans les espaces très confinés : quelques exemples
}

\author{
par A. Boudia $\left({ }^{1}\right)$, R. Gaudemer, A. Ouibrahim $\left({ }^{2}\right)$, D.H. Fruman \\ Groupe Phénomènes d'Interface \\ Ecole Nationale Supérieure de Techniques Avancées
}

\section{I — VALVE DE DIRECTION ASSISTÉE}

Une valve de direction assistée est composée d'une chemise (distributeur) fixe, comportant des conduites d'alimentation et d'évacuation, et d'un rotor (tiroir rotatif), solidaire d'une barre de torsion et d'un pignon engrenant avec une crémaillère. La barre de torsion se déforme angulairement, d'un angle maximum de $\pm 3^{\circ}$, et proportionnellement au couple appliqué au volant. De ce fait, il se produit un décalage angulaire entre la chemise et le rotor et la modification des sections de passage de l'huile, véhiculée au moyen d'une pompe par l'intermédiaire d'un régulateur. Cela conduit à l'augmentation de la pression qui s'exerce sur le piston d'un vérin qui entraîne, à son tour, la crémaillère. La force produite par le vérin réduit proportionnellement l'effort du conducteur. Lorsque celui-ci maintient le volant à un angle donné, le système retrouve une position d'équilibre diffé- rente de la position correspondant à la conduite en ligne droite. En effet, la barre de torsion, en se déformant sous le couple, décale le rotor par rapport à la chemise faisant admettre l'huile dans la chambre adéquate du vérin. Cette position rotor/chemise assure l'équilibre entre l'effort fourni par le conducteur au volant, celui dû au vérin et l'effort de rappel aux roues dû à la géométrie du train avant et à la sollicitation des pneumatiques.

(1) Adresse actuelle : Delphi, Saginaw Steering Systems, 95972 Roissy CDG Cedex.

Le travail sur les valves de direction assistée a été réalisé par Monsieur Boudia dans le cadre d'une bourse financée par la Direction de la Recherche de Renault.

(2) Adresse actuelle : Institut de Génie Mécanique, Université Mouloud Mammeri, Tizi-Ouzou, Algérie.

\section{Cavitation in very confined spaces : some examples}

By a simplified definition, cavitation occurs when the local pressure is equal or smaller than the vapour pressure of the flowing liquid, regardless of the dimension of the domain occupied by the liquid. Generally, in internal or external hydrodynamics, the domains are very large as compared to the zones where cavitation can exist. However, there is a certain number of situations in internal hydrodynamics where the liquid is confined between fixed or moving walls limiting spaces of very small dimensions, of the order of $10^{-6}$. $10^{-5} \mathrm{~m}$. Typical examples of such situations are those encountered in lubrication, flow (or pressure) control by section restrictors, etc. The Groupe Phénomènes d'Interface of ENSTA has been involved for many years in research work on two areas of industrial application: one is related to the flow in control valves of car power steering systems and the other to the flow occurring in the vicinity of the contact between a rotating cylinder or sphere with a fixed flat or curved wall. The purpose of this communication is to present some experimental results on the cavitation phenomena in these two flow configurations. 
Les espaces confinés, définis par les surfaces extérieure du rotor et intérieure de la chemise, peuvent être considérés comme bidimensionnels puisque leur largeur est beaucoup plus grande que les dimensions caractérisant une section droite. On trouve fig. 1 , une chambre d'alimentation amont qui communique au travers d'un rétrécissement, soit avec une canalisation (côté $B$ ), définie par deux parois presque parallèles, soit directement (côté $A$ ) avec l'une des chambres d'évacuation. La hauteur de la fente, la hauteur et la longueur de la canalisation, dépendent de la position angulaire relative entre rotor et chemise. La hauteur des chambres est très grande par rapport à celle de la fente et de la canalisation.

L'écoulement à faible nombre de Reynolds dans les espaces confinés a été étudié par Boudia [1] au moyen d'un code numérique (STAR-CD ${ }^{\mathrm{TM}}$ ). L'espace constitué par une partie de la chambre d'alimentation, les rétrécissements et la chambre aval, a été représenté au moyen d'un maillage comportant 7594 cellules. La dimension la plus petite des mailles était de $0,3 \mu \mathrm{m}$. Le critère de convergence des calculs était que le résidu normalisé sur les vitesses soit inférieur à $10^{-3}$. Le nombre de Reynolds $R e$, défini en prenant comme longueur de référence la largeur équivalente de la conduite d'alimentation de section circulaire $l$, et comme vitesse de référence, la vitesse débitante dans la section d'entrée de surface $L \times l$, était compris entre 10 et 300 . Donc, le régime d'écoulement était laminaire pour toutes les conditions d'opération testées.

Les résultats ont mis en évidence que l'irruption du jet issu du rétrécissement dans la chambre aval donne lieu à l'établissement d'une zone de dépression sur la paroi de la chemise tout de suite en aval de la singularité. Quand le nombre de Reynolds augmente, deux décollements, sièges de faibles pressions, se produisent, l'un sur la chemise et l'autre sur le rotor. Finalement, quand $R e$ diminue, les zones de décollement ne peuvent plus être individualisées.

Les valeurs des coefficients de pression $C p$, définis avec $P_{r e f}$ égale à la pression à l'entrée de la chambre d'alimentation de la valve (fig. 1), ont été obtenues dans la totalité du domaine pour différents angles de valve et nombres de Reynolds. A partir de ces valeurs, on a déterminé la valeur minimale, $C p_{\min }$

$\mathrm{Si}$ la tension superficielle ne joue pas un rôle majeur dans l'équilibre des germes, la cavitation se produira si la pression locale minimale au sein du liquide est égale à $P_{v}$, pression de vapeur. A partir de la définition du coefficient de pression, l'apparition (disparition) de la cavitation se produira quand

$$
\sigma_{i, d}=-C p_{\min }
$$

où l'indice $i$ ou $d$ s'applique aux conditions d'apparition et de disparition respectivement.

\section{NOMENCLATURE}

$e$ entrefer

l largeur équivalente de la conduite d'alimentation de section circulaire

$\mathrm{L}_{\text {ref }}$ longueur de référence

$\mathrm{P}_{\text {ref }}$ pression de référence

$\mathrm{U}_{\text {ref }}^{n \text { vitesse de de référence }}$

$\mathrm{P}_{\mathrm{v}}$ pression de vapeur

$R$ rayon du cylindre

$\rho$ masse spécifique du liquide

$v$ viscosité du fluide

$\omega$ vitesse de rotation
Nombres sans dimension

Cp coefficient de pression

$$
C p=2 \frac{P-P_{r e f}}{\rho U_{r f f}^{2}}
$$

$\mathrm{Re}$

nombre de Reynolds

$$
R e=U_{r e f} L_{n e} / v
$$

nombre de cavitation

$$
\sigma=2 \frac{P_{r e f}-P_{v}}{\rho U_{r e f}^{2}}
$$

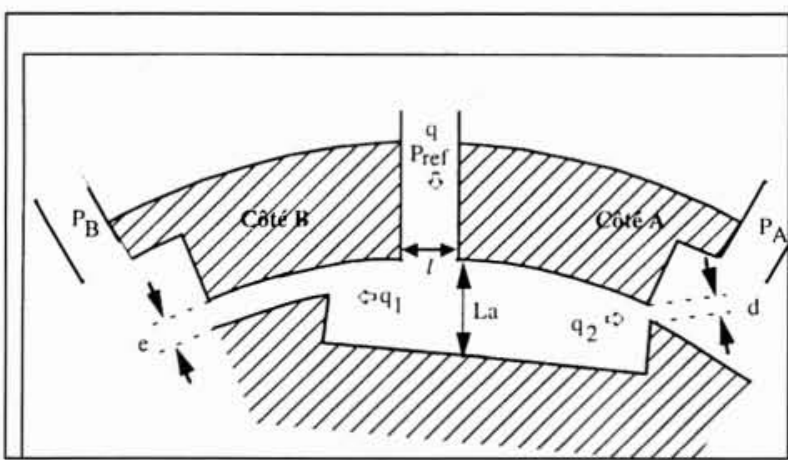

1. Schéma montrant les sections de passage d'huile dans la valve rotative.

( $L$ est la largeur des sections de passage perpendiculaire au plan de la figure).

Les conditions d'apparition (disparition) ont été déterminées en mesurant le bruit émis par la valve, placée dans une chambre anéchoïque de bruit de fond inférieur à $30 \mathrm{dBA}$, en fonction de l'angle, de la pression à l'entrée, du débit et de la température du liquide. Le mode opératoire était le suivant : après avoir atteint des conditions de fonctionnement stabilisées, on fixe :

- la température à l'entrée de la valve $\left(40^{\circ}, 60^{\circ}\right.$ ou $\left.80^{\circ} \mathrm{C}\right)$, - un angle de valve ( $\alpha$ varie de $0^{\circ}$ à $+3^{\circ}$ sachant que la valve est symétrique),

- un débit d'entrée et on fait varier la pression de référence grâce à une restriction en sortie de manière à transiter du régime non-cavitant au régime cavitant et vice-versa.

Puisque seuls les niveaux de bruit pour des angles de valve supérieurs à $2^{\circ}$ sont supérieurs à $30 \mathrm{dBA}$, niveau qui prévaut dans la chambre anéchoïque, les résultats sont présentés pour $\alpha=3^{\circ}$. La figure 2 montre que la modification de la température (donc, de la viscosité du liquide) donne lieu à un changement de la réponse en niveau de bruit. Puisque, pour ces différences de température, le changement de la pression de vapeur peut être négligé par rapport à la pression de référence, on doit admettre que le nombre de Reynolds a un effet non négligeable sur l'apparition de la cavitation. On choisit comme valeur de $\sigma_{i}$ celle correspondant au minimum des courbes de la figure 2 .

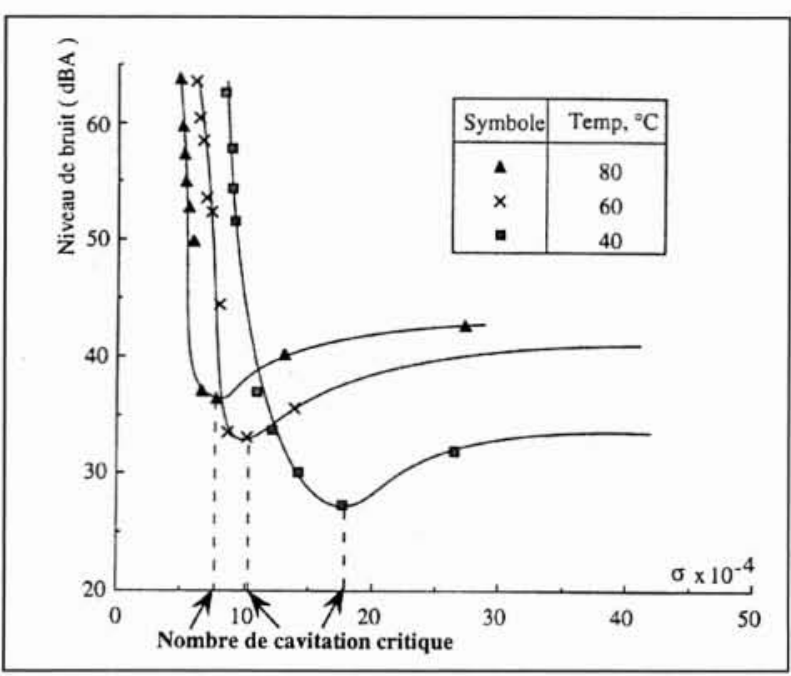

2. Niveau de bruit en fonction du nombre de cavitation. 
Le tableau 1 donne, pour chaque température du fluide, le $\sigma_{i}$, le Reynolds, ainsi que le $C p_{\min }$ obtenu au cours de la simulation numérique. Il est intéressant de constater que $\sigma_{i}$ est extrêmement proche, avec un écart maximum de $5 \%$, de $C p_{\min }$ (avec le signe changé).

Tableau 1. - Nombres de cavitation de naissance et coefficients de pression minimaux pour trois nombres de Reynolds et un angle de $3^{\circ}$.

\begin{tabular}{|c|c|c|c|}
\hline Température & $R e$ & $\sigma_{i}$ & $C p_{\min }$ \\
\hline $80^{\circ} \mathrm{C}$ & 42,7 & 78418 & -73975 \\
\hline $60^{\circ} \mathrm{C}$ & 26,5 & 100537 & -103379 \\
\hline $40^{\circ} \mathrm{C}$ & 12,2 & 176176 & -178660 \\
\hline
\end{tabular}

\section{II — LUBRIFICATION}

La diminution de la pression locale d'un liquide se trouvant dans un espace confiné par des parois mobiles peut conduire à ce qui est couramment appelé rupture du film. Les causes de ce phénomène peuvent être diverses. Downson [2], Downson et al. [3] et, plus récemment, Braun et Hendricks [4] distinguent celles associées au contenu en gaz dissous dans le liquide donnant lieu à la cavitation gazeuse (ou ventilation), celles résultant du changement de phase liquide/vapeur conduisant à la cavitation par vapeur (cavitation proprement dite) et, enfin, celles qui se manifestent quand la taille des bulles change à cause de la variation de la pression dans le film liquide (pseudo cavitation). La plupart des nombreuses recherches effectuées, Taylor [5], Cole et Hughes [6], Floberg [7, 8] et Downson et al. [3], concernent uniquement les conditions de ventilation. Cependant, celles donnant lieu à la cavitation sont pourtant réunies dans les paliers opérant à très grandes vitesses et complètement noyés dans des liquides, par exemple cryogéniques (Hydrogène ou Oxygène), à l'instar de ceux utilisés dans les turbopompes d'Ariane.

Les conditions géométriques (entrefers de dimensions micrométriques) et hydrodynamiques (forts gradients longitudinaux de pression et faibles, voire constants, gradients transversaux de pression) qui prévalent en lubrification dans des espaces très confinés sont telles qu'il a paru intéressant de conduire une recherche expérimentale destinée à établir les conditions de naissance et de développement de la cavitation. La première partie a été consacrée à l'étude d'une géométrie constituée par un cylindre en rotation au voisinage d'une paroi plane. Les résultats, présentés par Ouibrahim $e t$ al. $[9,10]$, ont mis en évidence que la cavitation se développait, dans cette géométrie, sous la forme d'un chapelet de minuscules poches dont la longueur maximale est inférieure au centimètre et la largeur maximale de l'ordre de $1,5 \mathrm{~mm}$. Les poches allongées (fig. 3) ont une forme pointue dirigée vers l'écoulement et sont séparées par un film liquide extrêmement mince. Les conditions de naissance de la cavitation et leur étendue azimutale ont été assez bien interprétées à partir des distributions de pression obtenues en fluide monophasique. On présente ici quelques résultats nouveaux concernant une géométrie sphère/paroi plane et sphère/paroi concave.

Le dispositif expérimental est constitué (fig. 4), d'une enceinte cylindrique en acier de $17,4 \mathrm{~cm}$ de diamètre intérieur et de $20,4 \mathrm{~cm}$ de hauteur. A l'intérieur de cette enceinte est placée, concentriquement, une sphère dont la zone équatoriale est en Téflon, de $7 \mathrm{~cm}$ de rayon $R$, pouvant atteindre une vitesse de rotation de 3000 tours/min. Le cylindre extérieur est muni d'une lucarne circulaire de $12 \mathrm{~cm}$ de diamètre

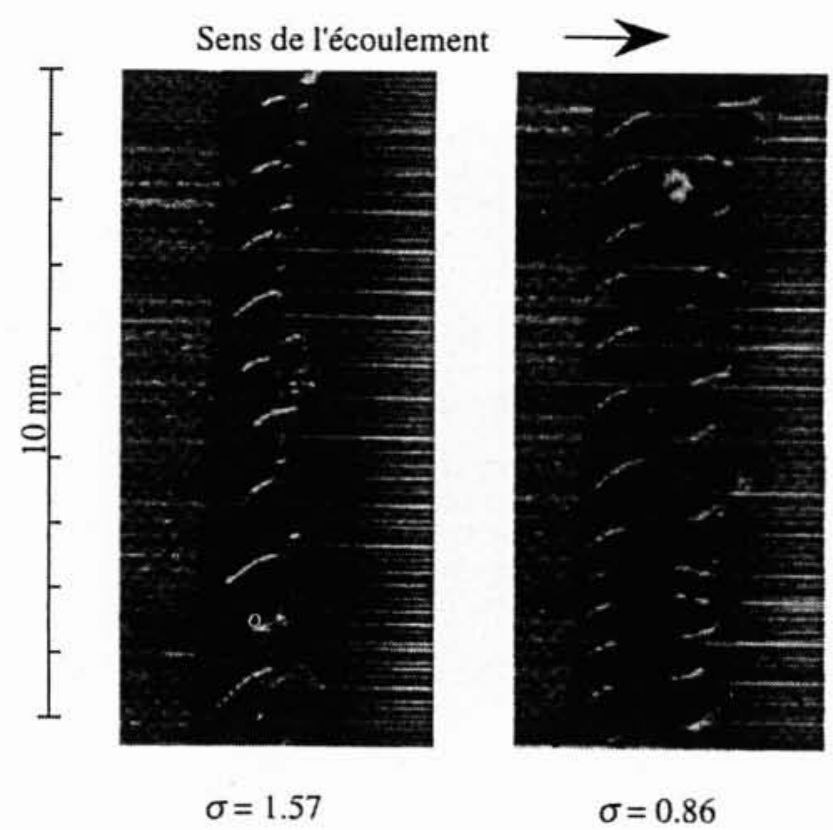

3. Cavitation développée dans un entrefer constitué par un cylindre en rotation et une paroi plane

Notez la forme des minuscules poches et leur régularité géométrique et spatiale.

à l'intérieur de laquelle coulisse un dispositif portant soit une paroi en verre à faces parallèles, soit une paroi dont la face interne est concave et épouse, avec un rayon de $7,28 \mathrm{~cm}$, la sphère. En déplaçant les parois en verre, perpendiculairement au plan tangent équatorial de la sphère, à l'aide de trois vis micrométriques, on modifie l'entrefer, $e$. Le liquide d'essai (eau) remplit totalement l'enceinte et est pompé au travers d'un échangeur de chaleur et d'un réservoir à surface libre au-dessus de laquelle on fait varier la pression. La pression de référence absolue ainsi que la pression différentielle de part et d'autre de l'espace confiné sont mesurées à l'aide de capteurs à membrane Validyne à partir de prises de pression disposées à mi-hauteur de l'enceinte de part et d'autre de la lucarne. Un thermomètre à lecture numérique inséré dans l'enceinte permet de relever les variations de température du liquide. Une caméra vidéo, disposée perpendiculairement à la paroi en verre, un magnétoscope et un moniteur TV permettent l'observation et l'enregistrement sur bande magnétique des phénomènes se produisant dans l'espace confiné. L'eau utilisée était, avant de procéder aux essais, dégazée par circulation sous vide et avec le cylindre tournant à faible vitesse de manière à éviter la rupture de film par dégazage.

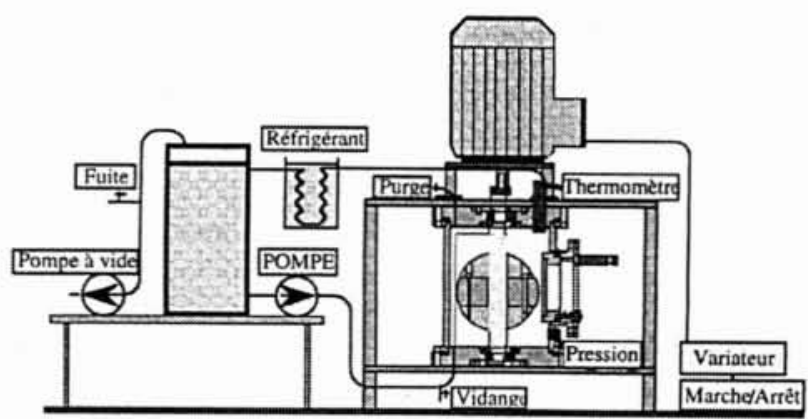

4. Schéma de la machine destinée à l'étude de la cavitation dans les entrefers constitués par une sphère en rotation et une paroi plane ou concave. 
a)
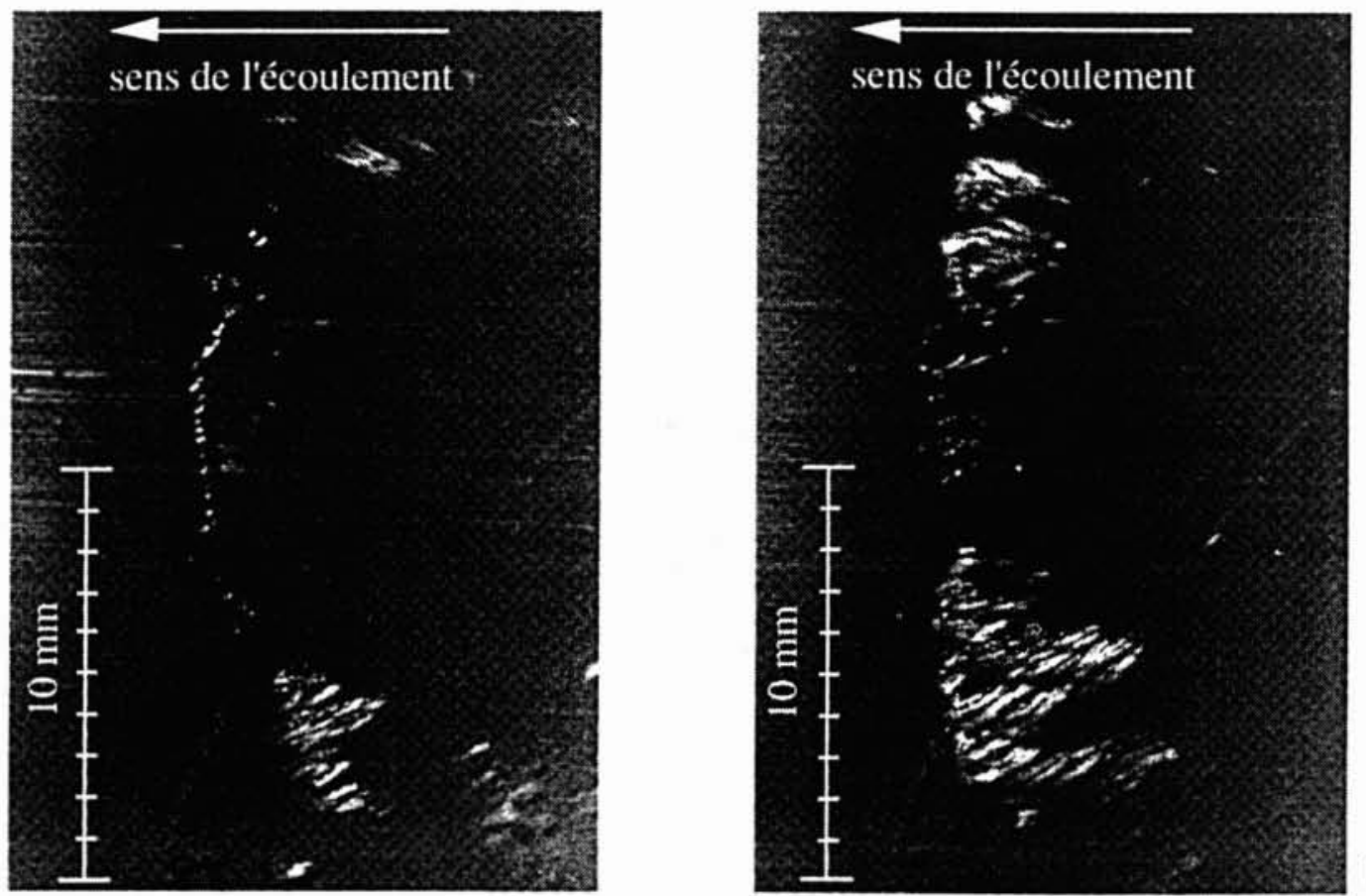

b)

5. Photographies de la cavitation dans les conditions de naissance pour $R e=99$ et $\sigma=0,46$ (a) et de la cavitation développée pour $\operatorname{Re}=123$ et $\sigma=0,3$ (b).

La figure 5 montre, dans le cas d'un entrefer minimum de $5 \mu \mathrm{m}$ entre la sphère et la paroi plane, les cavités qui se produisent pour une pression de référence de $74600 \mathrm{~Pa}$ et deux vitesses de référence. Pour une vitesse tangentielle de l'équateur de la sphère de $17,7 \mathrm{~m} / \mathrm{s}$, on a un début de cavitation (fig. $5 a$ ), avec quelques inclusions de très petites dimensions formant une ligne dans la zone où l'entrefer est faible et deux taches de plus grandes dimensions et plus diffuses de chaque côté. Quand la vitesse augmente, $22 \mathrm{~m} / \mathrm{s}$, la cavitation se développe, les taches deviennent plus importantes et se rapprochent (fig. $5 b$ ). On observe sur la figure $5 b$ des cavités ayant, comme dans le cas du cylindre, une forme pointue. Les photos, prises avec un stroboscope dont la durée de l'éclair est de $25 \mu \mathrm{s}$, montrent que les inclusions gazeuses latérales sont assez bien individualisées et sujettes à une composante de vitesse axiale importante. Les conditions mises en évidence sur la photo de la figure $5 a$ sont considérées comme étant celles d'apparition de la cavitation, celles de la photo présentée dans la figure $5 b$ comme correspondant à la cavitation développée.

La figure 6 présente les nombres de cavitation d'apparition $\sigma_{\vec{p}}$ calculés à partir de la vitesse $\omega R$, en fonction du nombre de Reynolds calculé avec l'entrefer $(e \omega R / v)$ pour un entrefer de $10 \mu \mathrm{m}$. A titre comparatif, on présente sur la même figure les nombres de cavitation $\sigma_{\text {id }}$ correspondant aux conditions de cavitation développée. On note que le $\sigma_{i}$ augmente avec le nombre de Reynolds, atteint un maximum et diminue ensuite. Pour les conditions de cavitation développée, comme dans le cas de l'apparition, les $\sigma_{i d}$ augmentent quand le nombre de Reynolds, relatif à l'entrefer, augmente, et atteignent ensuite un plateau sans qu'une diminution significative ne s'amorce. Etant donné que l'appréciation des conditions d'apparition est relativement subjective, l'expérience a été répétée trois fois pour s'assurer de la reproductibilité des résultats. Les tendances mises en évidence sur la figure 6 ont été confirmées.

L'effet de la distance minimale entre la sphère et la paroi a été étudié pour trois entrefers $(5,10$ et $20 \mu \mathrm{m})$. On voit (fig. 7) que l'ordre de grandeur des $\sigma_{i}$ reste pratiquement le même quel que soit l'entrefer. On note que les $\sigma_{i}$ se déplacent vers les nombres de Reynolds, relatifs à l'entrefer, plus faibles, pour les entrefers les plus petits.

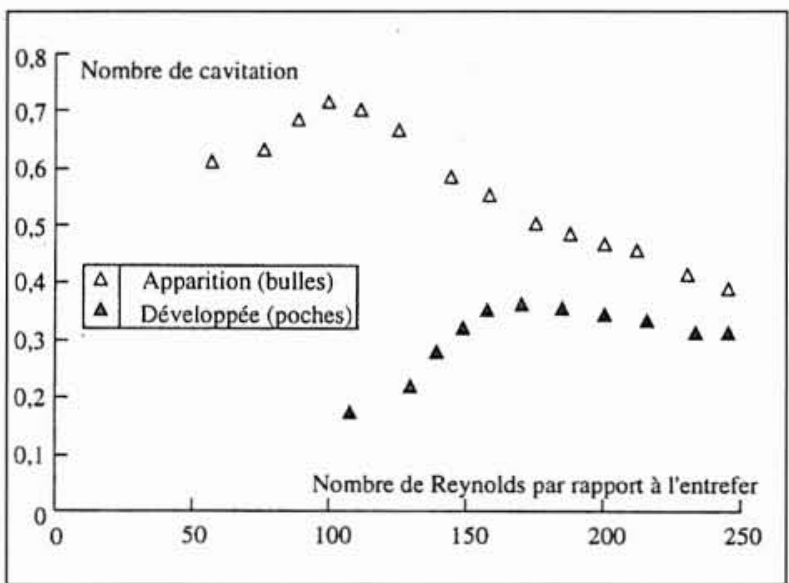

6. Nombre de cavitation pour les conditions d'apparition des bulles et d'une cavité développée en fonction du nombre de Reynolds par rapport à l'entrefer. Entrefer de $\mathbf{1 0} \mu \mathrm{m}$.

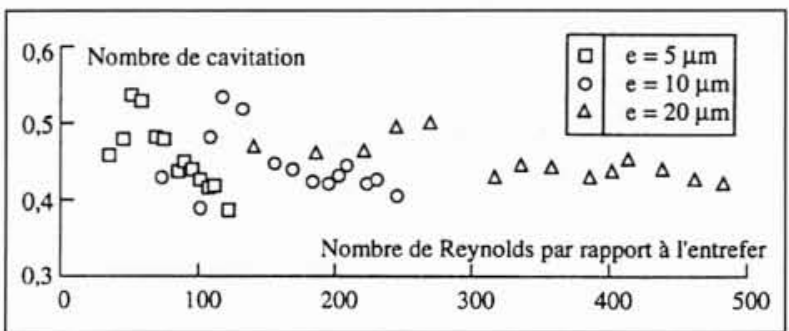

7. Nombre de cavitation d'apparition en fonction du nombre de Reynolds pour trois entrefers. 
Les conditions de cavitation développée pour trois entrefers sont présentées sur la figure 8. La modification de la distance entre la sphère et la paroi plane ne semble pas affecter significativement la valeur maximale atteinte par le nombre de cavitation mais produit un déplacement vers les nombres de Reynolds plus élevés quand l'entrefer est augmenté.

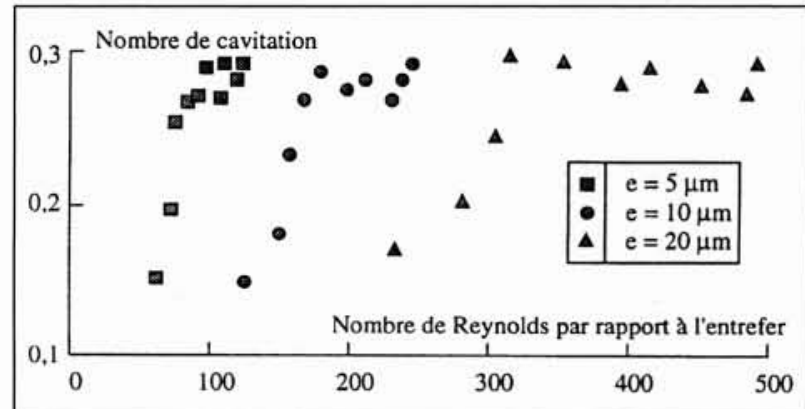

8. Nombre de cavitation pour l'apparition de la poche développée en fonction du nombre de Reynolds par rapport à l'entrefer, pour trois entrefers.

La figure 9, dans le cas de la sphère et de la paroi concave et pour un nombre de Reynolds de 148 (calculé avec l'entrefer minimum), montre l'apparition de la cavitation pour $\sigma=0,76$ et son développement ultérieur quand la pression (et donc le nombre de cavitation) diminue. A l'apparition de la cavitation, on note que des cavités isolées se situent de part et d'autre de l'entrefer minimum. Cela est dû au fait que, comme il a été vérifié en contrôlant les cotes du mobile, un aplatissement de la sphère dans la zone équatoriale s'était produit pendant des essais de contact. Quand on avance dans le développement de la cavitation, on voit les cavités isolées augmenter en nombre et en dimension et adopter une forme en pointe très proche de celle observée dans le cas des essais avec un cylindre et une paroi plane. Les conditions mises en évidence sur la figure 9, pour $\sigma=0,76$, sont considérées comme étant celles d'apparition de la cavitation.
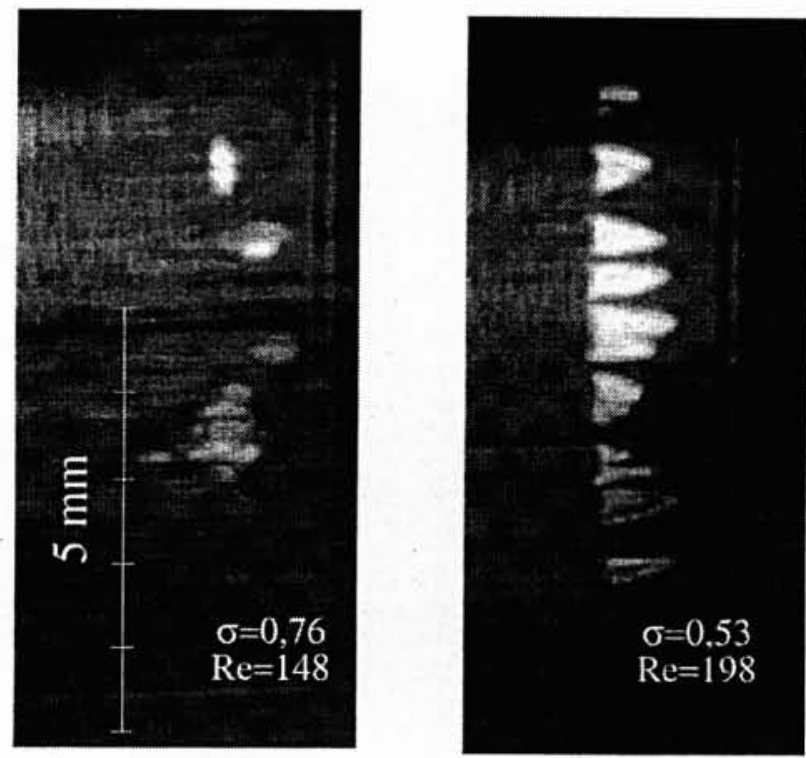

9. Photographies de la cavitation dans les conditions de naissance $(\sigma=0,76)$ et de cavitation développée $(\sigma=0,53)$ pour un entrefer de $\approx 10 \mu \mathrm{m}$. Direction de l'écoulement de droite à gauche.
La figure 10 présente les nombres de cavitation d'apparition $\sigma_{i}$, en fonction du nombre de Reynolds calculé avec l'entrefer. On note que, dans la gamme des nombres de Reynolds essayée, $\sigma_{i}$ diminue très légèrement avec $R e$. Les résultats sont tout à fait comparables à ceux obtenus avec la sphère et la paroi plane, reportés pour comparaison sur la même figure.

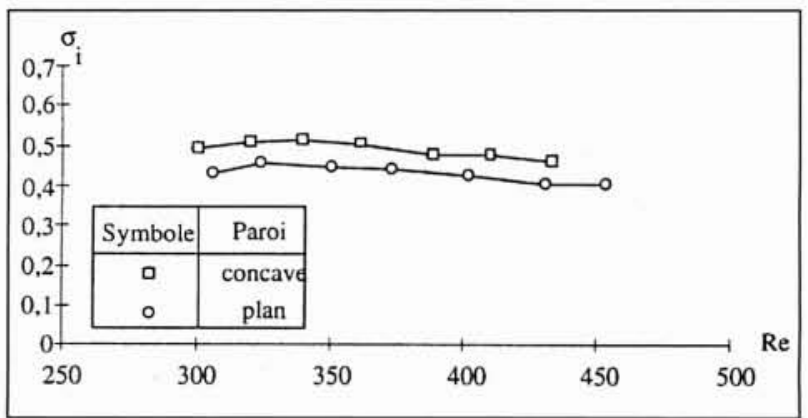

10. Nombre de cavitation d'apparition des bulles en fonction du nombre de Reynolds par rapport à l'entrefer. Cas de la sphère opérant avec une paroi plane et une paroi concave. Entrefer de $20 \mu \mathrm{m}$.

Les résultats obtenus mettent en évidence que les conditions d'apparition et de développement de la cavitation dans l'espace confiné limité par une sphère en rotation et une paroi plane sont totalement différentes de celles qui ont été mises en évidence dans une géométrie bidimensionnelle cylindre/paroi plane, où les nombres de cavitation d'apparition étaient inversement proportionnels au nombre de Reynolds calculé avec l'entrefer. Pour des entrefers et nombres de Reynolds comparables, les nombres de cavitation d'apparition sont, dans la situation sphère/plaque plane, inférieurs à ceux obtenus dans la géométrie cylindre/plaque plane. Par ailleurs, la modification de l'entrefer ne modifie pas significativement les valeurs maximales des nombres de cavitation critiques, qu'il s'agisse de l'apparition des premières inclusions ou de cavités développées.

Le fait que les nombres de cavitation critiques ne soient que peu influencés par la modification du nombre de Reynolds rapporté à l'entrefer, quel que soit celui-ci, donne à penser que la phénoménologie est dominée par des effets d'inertie et non pas par des effets visqueux. Pour donner une certaine justification à cette hypothèse, on a porté sur la figure 11 les nombres de cavitation critiques en fonction des nombres de Reynolds calculés en prenant, comme longueur de référence, le rayon de la sphère. On note que, en tenant compte de la dispersion associée à ces données expérimentales, la corrélation est satisfaisante. Pour le nombre de cavitation correspondant aux cavités développées, on observe aussi une corrélation analogue. Un tel comportement pourrait être expliqué en tenant compte du fait que l'écoulement au voisinage de l'entrefer minimum entre la sphère et la paroi plane a un caractère tridimensionnel accentué.

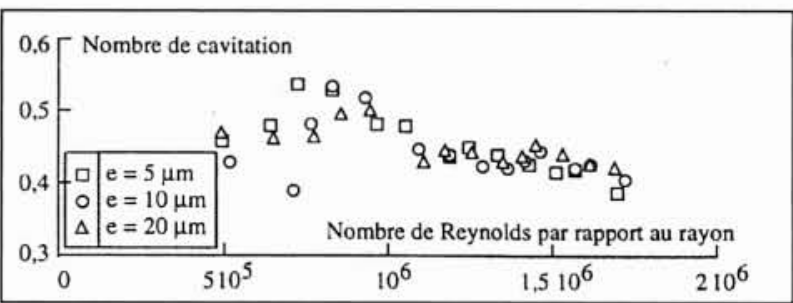

11. Nombre de cavitation d'apparition en fonction du nombre de Reynolds par rapport au rayon de la sphère, pour trois entrefers sphère/paroi plane. 
En ce qui concerne la géométrie sphère/paroi concave, les inclusions gazeuses sont géométriquement analogues à celles observées dans le cas du cylindre/paroi plane et s'étendent sur une distance très courte, de moins d'un dixième du rayon de la sphère, de chaque côté du point d'entrefer minimum. Leur apparition et leur développement ultérieur semblent aussi être déterminés par des phénomènes inertiels, comme dans la géométrie sphère/paroi plane. Ce type de comportement devrait être confirmé par de nouveaux essais avec des entrefers plus petits.

\section{III —CONCLUSION}

Les deux exemples présentés montrent l'importance des phénomènes de cavitation dans des écoulements ayant lieu dans des espaces confinés de dimensions micrométriques, avec ou sans parois mobiles. En particulier, dans le cas d'une valve de direction assistée, nos études ont permis de montrer que la prévision, au moyen d'un code de calcul Navier-Stokes, du coefficient de pression minimum dans le domaine fluide permettait de prévoir, comme il a été vérifié expérimentalement, les nombres de cavitation d'apparition. Dans le cas des écoulements de lubrification simulant les géométries existant au contact des billes avec les pistes des roulements à billes, on a mis en évidence l'évolution des nombres de cavitation critiques (apparition et développement) avec le nombre de Reynolds défini avec, comme échelle caractéristique, le rayon de la sphère en rotation.

\section{REMERCIEMENTS}

Les travaux sur la cavitation en lubrification de roulements noyés ont été conduits grâce au financement de l'Agence Française de l'Espace (CNES), dans le cadre des actions du GRT « Contacts Mécaniques ». Les auteurs remercient Messieurs Gérard Albano et Jean-François Dupuis, du CNES, pour la confiance dont ils ont bénéficié. Monsieur Ahmed Ouibrahim remercie l'ENSTA de lui avoir permis de contribuer à ce travail en tant que Professeur Invité. Le travail de Monsieur A. Boudia a été possible grâce au soutien de la Direction de la Recherche de Renault et au suivi attentif de Madame Pascale Minier.

\section{RÉFÉRENCES}

[1] Boudia A. (1995). - « Hydraulique et énergétique de la direction assistée d'un véhicule automobile ", Thèse, Université Paris VI.

[2] Dowson D. (1968). - " Cavitation in Lubricating Films ", Tribology, $1, \mathrm{~N}^{\circ} 3$, pp. 153-156

[3] Dowson D., Sмith E.H., Taylor C.M. (1976). — «A New Model for the Rupture of the Lubricating Film in Finite-Width Plain Bearings n, Proc. Inst. Mech. Eng. Tribology, pp. 1-6.

[4] BRAUN M.J., HENDRICKS R.C. (1984). - * An Experimental Investigation of the Vaporous/Gaseous Cavity Characteristics of an Eccentric Journal Bearing ", ASLE Transactions, Vol. 27, 1, pp. 1-14.

[5] TAYLOR G.I. - "Cavitation of a Viscous Fluid in Narrow Passages n, from The Scientific Papers of Sir G. I. Taylor, Vol. IV paper $\mathrm{N}^{\circ} 38$, (1963), edited by G. K. Batchelor, Cambridge University Press (1971)

[6] Cole J.A., Hughes C.J. (1956). - « Oil Flow and Film Extent in Complete Journal Bearings ", Proc. Inst. Mech. Eng. 170 , pp. $499-510$.

[7] Floberg L. (1961). - « Experimental Investigation of Cavitation Regions in Journal Bearings ", Report $\mathrm{N}^{\circ} 16, \mathrm{Nr}$ 238, Institute of Machine Elements, Chalmers University of Technology, Gothenburg, Sw.

[8] Floberg L. (1965), - « On Hydrodynamic Lubrication with Special Reference to Sub-cavity Pressures and Number of Streamers in Cavitation Regions ", Acta Polytechnica Scandinavica, Mech. Eng. Series N 19, Stockholm.

[9] Ouibrahim A., Fruman D.H., Gaudemer R. (1995). "Vapour Cavitation in Confined Spaces ", ASME FED 210, pp. 105-109.

[10] Ouibrahim A., Fruman D.H., Gaudemer R. (1996). " Vapour Cavitation in Very Confined Spaces for Newtonian and non Newtonian Fluids ", ASME FED 210, pp. 105-109 (1995). Physics of Fluids, 8 [7], pp. 1964-1971. 